\title{
An Energy Dissipative Spatial Discretization for the Regularized Compressible Navier-Stokes-Cahn-Hilliard System of Equations*
}

\author{
Vladislav Balashov ${ }^{a}$ and Alexander Zlotnik ${ }^{b, a}$ \\ ${ }^{a}$ Keldysh Institute of Applied Mathematics \\ Miusskaya sqr., 4, 125047 Moscow, Russia \\ ${ }^{b}$ National Research University Higher School of Economics \\ Pokrovskii bd. 11, 109028 Moscow, Russia \\ E-mail(corresp.): azlotnik@hse.ru \\ E-mail: vladislav.balashov@gmail.com
}

Received July 2, 2019; revised November 19, 2019; accepted November 22, 2019

\begin{abstract}
We consider the regularized 3D Navier-Stokes-Cahn-Hilliard equations describing isothermal flows of viscous compressible two-component fluids with interphase effects. We construct for them a new energy dissipative finite-difference discretization in space, i.e., with the non-increasing total energy in time. This property is preserved in the absence of a regularization. In addition, the discretization is well-balanced for equilibrium flows and the potential body force. The sought total density, mixture velocity and concentration of one of the components are defined at nodes of one and the same grid. The results of computer simulation of several $2 \mathrm{D}$ test problems are presented. They demonstrate advantages of the constructed discretization including the absence of the so-called parasitic currents.
\end{abstract}

Keywords: two-component two-phase isothermal flows, interphase effects, regularized viscous compressible Navier-Stokes-Cahn-Hilliard equations, finite-difference discretization in space, energy dissipativeness.

AMS Subject Classification: 65M06; 35Q35.

\footnotetext{
* This article was originally published with error. Please see Corrigendum https://doi.org/10.3846/mma.2021.14527
}

Copyright (c) 2020 The Author(s). Published by VGTU Press

This is an Open Access article distributed under the terms of the Creative Commons Attribution License (http://creativecommons.org/licenses/by/4.0/), which permits unrestricted use, distribution, and reproduction in any medium, provided the original author and source are credited. 


\section{Introduction}

Multiphase microflows involving significant interphase and capillary effects are often appear in nature and technology. Development and improvement of relevant mathematical models and numerical methods for their implementation is an important and urgent task. One of the common models for describing isothermal flows of two-component two-phase mixtures with the direct resolution of the interface and taking into account the associated effects is the Navier-Stokes-Cahn-Hilliard (NSCH) system of equations. It can be attributed to the group of the phase field models (methods of the diffuse interface type). In the frame of such models, the interface is actually a layer of small but finite thickness within which interphase forces (for example, related to the surface tension) operate. In this case, the spatial distribution of the phases is described by the distribution of an order parameter which is usually concentration and/or density. Accounting for interphase effects is provided, on the one hand, by introducing the dependence of the Helmholtz free energy (or another thermodynamic potential) on the modulus of the order parameter gradient and, on the other hand, by its non-convex dependence on the order parameter. For more details, for example, see $[2,19,20]$.

Notice that in such a model the concept of a phase is not explicitly introduced. However, owing to the mentioned special form of the free energy, in the flow domain, some subdomains arise that are occupied by a mixture with an almost homogeneous composition in space, i.e., actually by a separate phase.

In this paper, we consider a special quasi-hydrodynamic (QHD) regularization of the NSCH equations [3] consisting in a thermodynamically justified introducing of some terms with a small parameter into the original equations. They are able to play the role of numerical regularizers allowing to apply relatively simple (conditionally) stable and convenient in parallelization of computations explicit central finite-difference schemes [4,5]. Previously, the QHD and related quasi-gas-dynamic approaches to regularization were successfully applied to various gas dynamics systems of equations including the case of binary mixtures; moreover, the introduced terms allow one to improve matching of numerical results with experimental data for moderately rarefied gas flows, see $[7,10,11,21]$. Note that there exist other recent approaches to the regularization of the gas dynamics equations, in particular, see $[13,22]$.

In the numerical simulation of multiphase flows using the direct resolution of the interface and taking into account related effects, the so-called spurious, or parasitic, currents arise frequently. This purely numerical phenomenon means a vortex distribution of the velocity field in the vicinity of the interface which does not decay in time. This phenomenon is inherent not only for numerical methods to solve the NSCH system of equations (or other phase field models) but also for other kind methods such as the volume of fluid method [14], the level set method and the lattice Boltzmann method. Often, the parasitic currents have a significant negative impact on the flow dynamics. Depending on the mathematical model underlying the specific method, various methods of dealing with them are applied $[1,8,14,16]$.

For the models of phase-field type, the absence of conservativeness in the 
total energy (in the sense of the mutual balance between the free energy of the system and the kinetic energy associated with surface forces) at the discrete level was indicated as the cause of the parasitic currents in $[15,16]$. In particular, it seems that the fulfillment of such a property together with adequate symmetry properties and the positive definiteness of the Navier-Stokes viscous stress discrete operator imply the energy dissipativity of the finite-difference scheme (in the sense of non-increasing of its total energy). To ensure such a balance (thus the dissipativity as well), it was also proposed there to use a special non-divergent form of the momentum equation while constructing finite-difference schemes. On the other hand, strictly speaking, then the conservativeness in momentum is normally lost although being still satisfied within the approximation error. However, for a wide range of problems, it seems that the preservation of the energy dissipativity at the discrete level is much more important.

This paper is devoted to the construction of a new energy-dissipative finitedifference spatial discretization for the 3D QHD-regularized viscous compressible isothermal NSCH system of equations taking into account the potential body force, in the spatially periodic statement. This construction is based, firstly, on the recent dissipative discretizations of regularized barotropic and full 3D gas dynamics systems of equations from $[26,27]$ and, secondly, on the representation in the potential form of a term associated to the capillary forces in the momentum equation similarly to $[15,16,17]$. In this discretization, the sought functions, namely, the density and velocity of the mixture and the concentration of one of the components are defined at nodes of one and the same grid. The corresponding 1D construction has recently been accomplished in [6]. The constructed 3D discretization is additionally well-balanced for equilibrium solutions and the potential body force.

Strictly speaking, the constructed discretization is not conservative in momentum as in the indicated papers. But in simulations performed in this paper we observe the preservation of the total momentum of the system within the high precision $\sim 10^{-14}-10^{-20}$.

The paper is organized as follows. In Section 2, the regularized NSCH system of equations is presented. For the system, the total energy balance equation and the potential form of the momentum equation are given. In Section 3, we construct a new spatial discretization of the equations and prove that its total energy is non-increasing in the spatially periodic statement of the problem. In addition, we prove that the constructed discretization is wellbalanced for the potential body force. Also a version of this discretization more close to [6] is discussed. The last Section 4 is devoted to computer simulation of three 2D test problems. We apply the explicit Euler approximation in time and compare the numerical results obtained by the schemes constructed in this paper and previously in [5] and emphasize advantages of the new schemes, in particular, the absence of parasitic currents for them: the maximum value of the velocity modulus is zero within the computer precision when the solution stabilizes. We also give some negative numerical results in the case where the regularization is absent. 


\section{The regularized Navier-Stokes-Cahn-Hilliard equations}

We consider the Navier-Stokes-Cahn-Hilliard (NSCH) system of equations describing isothermal viscous compressible flows of two-phase two-component mixture and taking into account interphase (surface) effects $[2,19,20]$. Its regularized version $[3,4,5]$ consists of the total mass, momentum and component mass balance equations

$$
\begin{gathered}
\partial_{t} \rho+\operatorname{div} \mathbf{J}=0, \\
\partial_{t}(\rho \mathbf{u})+\operatorname{div}(\mathbf{J} \otimes \mathbf{u})+\nabla p(\rho, C)=\operatorname{div} \mathbf{\Pi}+\rho \nabla \Phi, \\
\partial_{t}(\rho C)+\operatorname{div}(\mathbf{J} C)=\operatorname{div}[M(C) \nabla \mu(\rho, C, \nabla C)],
\end{gathered}
$$

where $\rho>0$ is the total density, $\mathbf{u}=\left(u_{1}, u_{2}, u_{3}\right)$ is the velocity of both mixture components, $0<C<1$ is the mass concentration of one of two considered components all depending on $(x, t)$, with $x=\left(x_{1}, x_{2}, x_{3}\right) \in \mathbb{R}^{3}$ and $t \geqslant 0$. Hereafter $\partial_{t}=\partial / \partial t$ and $\partial_{i}=\partial / \partial x_{i}$, the divergence of a tensor is taken with respect to its first index, symbols $\otimes$ and $\cdot$ correspond respectively to the tensor and inner products of vectors. In addition, $\Phi(x)$ is the potential of a stationary body force and $M(C)>0$.

We impose the condition of $X:=\left(X_{1}, X_{2}, X_{3}\right)$ - periodicity of the solution and the function $\Phi(x)$ with respect to $\left(x_{1}, x_{2}, x_{3}\right)$, where $X_{i}>0, i=1,2,3$. We set $\Omega_{X}:=\left(0, X_{1}\right) \times\left(0, X_{2}\right) \times\left(0, X_{3}\right)$.

The regulirized mass flux $\mathbf{J}$ is given by the formulas

$$
\mathbf{J}=\rho \mathbf{u}-\mathbf{m}, \quad \mathbf{m}=\tau[\rho(\mathbf{u} \cdot \nabla) \mathbf{u}+\nabla p-\rho \nabla \Phi+\operatorname{div} \mathbf{Q}] .
$$

It includes the regularizing momentum $\mathbf{m}$ with the regularization parameter $\tau=\tau(\rho, \mathbf{u}, C)>0$; following [25], we use $\mathbf{m}$ not the regularizing velocity.

The tensor $\boldsymbol{\Pi}=\boldsymbol{\Pi}^{N S}-\mathbf{Q}+\boldsymbol{\Pi}^{\tau}$ consists of the Navier-Stokes viscous stress tensor $\boldsymbol{\Pi}^{N S}$, the capillary stress tensor $\mathbf{Q}$ and the regularizing tensor $\boldsymbol{\Pi}^{\tau}$ :

$$
\begin{array}{cc}
\mathbf{\Pi}^{N S}=2 \eta \mathbf{D}(\mathbf{u})+\left(\zeta-\frac{2}{3} \eta\right)(\operatorname{div} \mathbf{u}) \mathbf{I}, & \mathbf{D}(\mathbf{u})=\frac{1}{2}\left(\nabla \mathbf{u}+\nabla \mathbf{u}^{T}\right), \\
\mathbf{Q}=\lambda_{1} \rho \nabla C \otimes \nabla C, & \mathbf{\Pi}^{\tau}=\mathbf{u} \otimes \mathbf{m},
\end{array}
$$

where $\nabla \mathbf{u}=\left\{\partial_{i} u_{j}\right\}_{i, j=1}^{3}, \eta=\eta(\rho, C)>0$ and $\zeta=\zeta(\rho, C) \geq 0$ are the coefficients of dynamic and bulk viscosity as well as $\mathbf{I}$ is the identity tensor.

The regularizing terms in the equations are physically based $[3,7,10,21]$. In what follows, they provide (conditional) stability of explicit central finitedifference approximations of the equations.

It remains to define the pressure $p$ and the (generalized) chemical potential $\mu$. According to [18] we set the Helmholtz free energy of the mixture as follows

$$
\begin{gathered}
\Psi(\rho, C, \nabla C)=\Psi_{0}(\rho, C)+\frac{1}{2} \lambda_{1}|\nabla C|^{2} \\
\Psi_{0}(\rho, C)=C c_{s 1}^{2} \ln \frac{\rho}{\bar{\rho}_{1}}+(1-C) c_{s 2}^{2} \ln \frac{\rho}{\bar{\rho}_{2}}+\Psi_{s e p}(C),
\end{gathered}
$$

where the constant $\lambda_{1}>0$ is a model parameter, $c_{s i}$ and $\bar{\rho}_{i}>0$ are the sound speed and reference density of $i$ th component. There exist various ways to set 
the function $\Psi_{\text {sep }}(C)$. According to $[2,18,19]$, we apply the following polynomial form

$$
\Psi_{\text {sep }}(C)=A_{\psi} C^{2}(1-C)^{2}, \quad A_{\psi}>0,
$$

which is also used in numerical simulations below in Section 4. The presence of two local minima at $C=0,1$ corresponding to "pure" components is peculiar to $\Psi_{\text {sep }}(C)$ that is of great concern in simulation of interphase (surface) effects.

The functions $p$ and $\mu$ are introduced due to $\Psi_{0}(\rho, C)$ :

$$
\begin{aligned}
& p(\rho, C)=\rho^{2} \Psi_{0 \rho}^{\prime}(\rho, C)=c_{s 1}^{2} \rho C+c_{s 2}^{2} \rho(1-C), \\
& \mu=\mu(\rho, C, \nabla C)=\Psi_{0 C}^{\prime}(\rho, C)-\frac{\lambda_{1}}{\rho} \operatorname{div}(\rho \nabla C) .
\end{aligned}
$$

Hereafter $(\cdot)_{\rho}^{\prime}=\partial / \partial \rho$ and $(\cdot)_{C}^{\prime}=\partial / \partial C$. It should be noted that the nonlinear terms with $\nabla C$ appearing in $\mathbf{Q}$ in combination with the non-convexity in $C$ of $\Psi_{0}$ are responsible for the surface effects.

If $\tau=0$, then $\mathbf{m}=0$, and system (2.1)-(2.3) becomes the standard NSCH system for viscous compressible two component isothermal mixture flows.

We supplement the system (2.1)-(2.3) with the initial conditions:

$$
\left.\rho\right|_{t=0}=\rho_{0}(x),\left.\quad \mathbf{u}\right|_{t=0}=\mathbf{u}_{0}(x),\left.\quad C\right|_{t=0}=C_{0}(x)
$$

with the $X$-periodic initial functions $\rho_{0}, \mathbf{u}_{0}$ and $C_{0}$.

The following total mass and component mass conservation laws hold

$$
\int_{\Omega_{X}} \rho(x, t) d x=\int_{\Omega_{X}} \rho_{0}(x) d x, \quad \int_{\Omega_{X}}(\rho C)(x, t) d x=\int_{\Omega_{X}}\left(\rho_{0} C_{0}\right)(x) d x
$$

for $t \geq 0$.

For the considered system, the following result is also valid [5].

Proposition 1. Let $e=\Psi_{0}(\rho, C)+\frac{1}{2} \lambda_{1}|\nabla C|^{2}+\frac{1}{2}|\mathbf{u}|^{2}-\Phi$ be the specific full energy. For the total energy $\mathcal{E}:=\int_{\Omega_{X}} \rho e d x$, the following balance equation holds

$$
\partial_{t} \mathcal{E}+\int_{\Omega_{X}}\left[2 \eta|\mathbf{D}(\mathbf{u})|^{2}+\left(\zeta-\frac{2}{3} \eta\right)(\operatorname{div} \mathbf{u})^{2}+M|\nabla \mu|^{2}+\frac{1}{\rho \tau}|\mathbf{m}|^{2}\right] d x=0
$$

for $t \geq 0$. Here $2 \eta|\mathbf{D}(\mathbf{u})|^{2}+\left(\zeta-\frac{2}{3} \eta\right)(\operatorname{div} \mathbf{u})^{2} \geq 0,|\mathbf{D}(\mathbf{u})|^{2}=\mathbf{D}(\mathbf{u}): \mathbf{D}(\mathbf{u})$ is the inner product of the tensor with itself.

Consequently, the total energy is non-increasing in time: $\partial_{t} \mathcal{E} \leq 0$ for $t \geq 0$.

This result is essential and justifies the physical correctness of the above regularization. Note that the term with $\mathbf{m}$ disappears for $\tau=0$.

In [6], to construct an energy dissipative 1D discretization, the following non-divergent form of terms $\nabla p+\operatorname{div} \mathbf{Q}$ in equation (2.2) and formula (2.4) has recently been applied

$$
\begin{gathered}
\nabla p+\operatorname{div} \mathbf{Q}=\rho \nabla G-\rho \mu \nabla C, \\
G \equiv G(\rho, C, \nabla C):=\Psi_{1 \rho}^{\prime}(\rho, C)+\frac{1}{2} \lambda_{1}|\nabla C|^{2} .
\end{gathered}
$$


The same equality was given in [19]. Here $\Psi_{1}(\rho, C):=\rho \Psi_{0}(\rho, C)$ is the homogeneous (i.e., independent of $\nabla C$ ) Helmholtz free energy per unit volume. The function $G(\rho, C, \nabla C)=\Psi_{0}+\rho \Psi_{0 \rho}^{\prime}+\frac{1}{2} \lambda_{1}|\nabla C|^{2}=\Psi+\rho \Psi_{\rho}^{\prime}$ can be regarded as the mass density of the Gibbs free energy, and $\Psi_{1 \rho}^{\prime}$ is its homogeneous part (similarly to $\Psi_{0}$ in $\Psi$ ). In the next section, formulas $(2.9)-(2.10)$ are essentially used.

In addition, we consider the equilibrium solutions $\rho=\rho_{S}(x)>0, \mathbf{u}=\mathbf{u}_{S}=$ 0 and $0<C=C_{S}(x)<1$ of the considered system taking into account (2.9)(2.10). We assume that they also are $X$-periodic, and the system is reduced to the following form

$$
\operatorname{div} \mathbf{J}_{S}=0, \quad \nabla(G-\Phi)-\mu \nabla C=0, \quad \operatorname{div}\left(\mathbf{J}_{S} C-M(C) \nabla \mu\right)=0
$$

in $\mathbb{R}^{3}$, where $\mathbf{J}_{S}=\tau_{0} \rho[\nabla(G-\Phi)-\mu \nabla C]$ and $\tau_{0}=\tau(\rho, 0, C)$. It implies that $\mathbf{J}_{S}=0$, and the equations become the same as for the non-regularized system (for $\tau=0$ ). Therefore the above regularized system does not change equilibrium solutions of the non-regularized one.

The last equation in (2.11) is reduced to $\operatorname{div}(M(C) \nabla \mu)=0$ thus $\mu \equiv \mu_{S}=$ const. Then from the second equation in (2.11) we get $G-\mu_{S} C-\Phi \equiv$ const. Thus the equilibrium solutions $\rho_{S}$ and $C_{S}$ obey the following system in $\mathbb{R}^{3}$

$$
\begin{gathered}
\Psi_{1 \rho}^{\prime}(\rho, C)+\frac{1}{2} \lambda_{1}|\nabla C|^{2}-\mu_{S} C-\Phi \equiv \mathrm{const}, \\
\Psi_{1 C}^{\prime}(\rho, C)-\lambda_{1} \operatorname{div}(\rho \nabla C)=\mu_{S} \rho .
\end{gathered}
$$

\section{An energy dissipative spatial discretization of the regularized Navier-Stokes-Cahn-Hilliard system}

2.1. We first define the notation. We introduce the main uniform grid $\omega_{k h}$ in $x_{k} \in \mathbb{R}$ with nodes $x_{k m}=m h_{k}, m \in \mathbb{Z}, h_{k}=X_{k} / N_{k}$ and the auxiliary grid $\omega_{k h}^{*}$ with nodes $x_{k(m+1 / 2)}=(m+0.5) h_{k}, m \in \mathbb{Z}, k=1,2,3$. Let $H_{X_{k}}\left(\omega_{k h}\right)$ and $H_{X_{k}}\left(\omega_{k h}^{*}\right)$ be the spaces of $X_{k}$-periodic functions defined on these grids, endowed respectively with the inner products

$$
(v, \tilde{v})_{\omega_{k h}}=\sum_{m=0}^{N_{k}-1} v_{m} \tilde{v}_{m} h, \quad(y, \tilde{y})_{\omega_{k h}^{*}}=\sum_{m=1}^{N_{k}} y_{m-1 / 2} \tilde{y}_{m-1 / 2} h .
$$

For $v \in H_{X_{k}}\left(\omega_{k h}\right)$ and $y \in H_{X_{k}}\left(\omega_{k h}^{*}\right)$, we introduce the grid averages and difference quotients

$$
\begin{aligned}
& \left(s_{k} v\right)_{m-1 / 2}=\frac{1}{2}\left(v_{m-1}+v_{m}\right), \quad \delta_{k} v_{m-1 / 2}=\frac{v_{m}-v_{m-1}}{h}, \\
& \left(s_{k}^{*} y\right)_{m}=\frac{1}{2}\left(y_{m-1 / 2}+y_{m+1 / 2}\right), \quad \delta_{k}^{*} y_{m}=\frac{y_{m+1 / 2}-y_{m-1 / 2}}{h} .
\end{aligned}
$$

Clearly $s_{k}, \delta_{k}: H_{X_{k}}\left(\omega_{k h}\right) \rightarrow H_{X_{k}}\left(\omega_{k h}^{*}\right)$ and $s_{k}^{*}, \delta_{k}^{*}: H_{X_{k}}\left(\omega_{k h}^{*}\right) \rightarrow H_{X_{k}}\left(\omega_{k h}\right)$.

For $v \in H_{X_{k}}\left(\omega_{k h}\right)$ and $y \in H_{X_{k}}\left(\omega_{k h}^{*}\right)$, it is easy to check the following formulas

$$
\left(\delta_{k}^{*} y, v\right)_{\omega_{k h}}=-\left(y, \delta_{k} v\right)_{\omega_{k h}^{*}}, \quad\left(s_{k}^{*} y, v\right)_{\omega_{k h}}=\left(y, s_{k} v\right)_{\omega_{k h}^{*}}
$$


which are extensively used below.

We introduce the 3D grids $\omega_{h}=\omega_{1 h} \times \omega_{2 h} \times \omega_{3 h}, \omega_{h}^{*}=\omega_{1 h}^{*} \times \omega_{2 h}^{*} \times \omega_{3 h}^{*}$ and the grid $\omega_{i *, h}$ that is obtained from $\omega_{h}$ by replacing the multiplier $\omega_{i h}$ with $\omega_{i h}^{*}$, $i=1,2,3$. Let $H_{X}\left(\omega_{h}\right)$ and $H_{X}\left(\omega_{h}^{*}\right)$ be the spaces of $X$-periodic in $x$ functions defined on $\omega_{h}$ and $\omega_{h}^{*}$, endowed respectively with the inner products

$$
(v, \tilde{v})=\left(\left((v \tilde{v}, 1)_{\omega_{1 h}}, 1\right)_{\omega_{2 h}}, 1\right)_{\omega_{3 h}}, \quad(y, \tilde{y})_{*}=\left(\left((y \tilde{y}, 1)_{\omega_{1 h}^{*}}, 1\right)_{\omega_{2 h}^{*}}, 1\right)_{\omega_{3 h}^{*}} .
$$

Let $(v, \tilde{v})_{i *}$ be the inner product obtained from $(v, \tilde{v})$ by substituting $(\cdot, 1)_{\omega_{i h}}$ with $(\cdot, 1)_{\omega_{i h}^{*}}$.

2.2. Now we discretize the regularized NSCH equations on the grid $\omega_{h}$ as follows

$$
\begin{aligned}
& \partial_{t} \rho+\delta_{i}^{*} J_{i}=0 \\
& \partial_{t}\left(\rho u_{l}\right)+\delta_{i}^{*}\left(J_{i} s_{i} u_{l}\right)+s_{l}^{*}\left[\left(s_{l} \rho\right) \delta_{l} G_{h}\right]=\delta_{i}^{*}\left(\Pi_{i l}^{N S}+\Pi_{i l}^{\tau}\right) \\
& \quad+s_{l}^{*}\left\{\left(s_{l} \rho\right)\left[\left(s_{l} \mu\right) \delta_{l} C+\delta_{l} \Phi\right]\right\} \\
& \partial_{t}(\rho C)+\delta_{i}^{*}\left(J_{i} s_{i} C\right)=\delta_{i}^{*}\left(M\left(s_{i} C\right) \delta_{i} \mu\right)
\end{aligned}
$$

where $l=1,2,3$ and

$$
\begin{aligned}
& G_{h}=\Psi_{1 \rho}^{\prime}(\rho, C)+\mathcal{E}_{\lambda}, \quad \mathcal{E}_{\lambda}=\frac{1}{2} \lambda_{1} s_{i}^{*}\left[\left(\delta_{i} C\right)^{2}\right], \\
& \mu=\frac{1}{\rho}\left[\Psi_{1 C}^{\prime}(\rho, C)-\delta_{i}^{*}\left(\lambda_{1}\left(s_{i} \rho\right) \delta_{i} C\right)\right] .
\end{aligned}
$$

The basic unknown functions are $\rho(\cdot, t), \mathbf{u}(\cdot, t), C(\cdot, t) \in H_{X}\left(\omega_{h}\right)$, with $\rho>0$ and $0<C<1$, for $t \geq 0$. The parameter $\tau=\tau(\rho, \mathbf{u}, C)>0$ and function $\Phi \in H_{X}\left(\omega_{h}\right)$ are given. Hereafter the summation from 1 to 3 is assumed over the repeated indices $i$ and $j$ (and only over them).

We discretize the components of regularized mass flux $J_{k}(x, t)$ on $\omega_{k *, h}$, namely, the terms with $\rho, \mathbf{u}$ and $\Phi$ according to [27, Section 3.4] and the terms with $C$ according to $[6]$ :

$$
\begin{aligned}
J_{k}= & \left(s_{k} \rho\right) s_{k} u_{k}-\mathrm{m}_{k}, \\
\mathrm{~m}_{k}= & \left(s_{k}(\tau \rho)\right)\left[\left(s_{k} u_{k}\right) \delta_{k} u_{k}+\delta_{k}\left(G_{h}-\Phi\right)-\left(s_{k} \mu\right) \delta_{k} C\right] \\
& +s_{k}\left[\tau \rho s_{l}^{*}\left(\left(s_{l} u_{l}\right) \delta_{l} u_{k}\right)+\tau \rho s_{m}^{*}\left(\left(s_{m} u_{m}\right) \delta_{m} u_{k}\right)\right],
\end{aligned}
$$

see (2.4), (2.9) and (2.10). Hereafter $(k, l, m)$ is any permutation of $(1,2,3)$.

The tensor $\Pi^{N S}$ entries, see (2.5), are also discretized on $\omega_{k *, h}$ according to $[26$, Section 2.2]

$$
\begin{aligned}
\Pi_{k k}^{N S} \equiv \Pi_{k k}^{N S}(\mathbf{u})= & {\left[s_{l}^{*} s_{m}^{*}\left(\frac{4}{3} \eta+\zeta\right)\right] \delta_{k} u_{k} } \\
& +s_{l}^{*}\left[\left(s_{m}^{*}\left(\zeta-\frac{2}{3} \eta\right)\right) s_{k} \delta_{l} u_{l}\right]+s_{m}^{*}\left[\left(s_{l}^{*}\left(\zeta-\frac{2}{3} \eta\right)\right) s_{k} \delta_{m} u_{m}\right], \\
\Pi_{k l}^{N S} \equiv \Pi_{k l}^{N S}(\mathbf{u})= & \left(s_{l}^{*} s_{m}^{*} \mu\right) \delta_{k} u_{l}+s_{l}^{*}\left[\left(s_{m}^{*} \mu\right) s_{k} \delta_{l} u_{k}\right], \quad k \neq l .
\end{aligned}
$$


The tensor $\boldsymbol{\Pi}^{\tau}$ entries, see (2.6), are discretized on $\omega_{k *, h}$ as in [27, Section $3.4]$

$$
\begin{gathered}
\Pi_{k k}^{\tau}=\left(s_{k} u_{k}\right) \mathrm{m}_{k}, \quad \Pi_{k l}^{\tau}=\left(s_{k} u_{k}\right) \mathrm{m}_{l}^{(k)}, \quad k \neq l \\
\mathrm{~m}_{l}^{(k)}=s_{k}\left\{\tau \rho s_{l}^{*}\left[\left(s_{l} u_{l}\right) \delta_{l} u_{l}+\delta_{l}\left(G_{h}-\Phi\right)-\left(s_{l} \mu\right) \delta_{l} C\right]+\tau \rho s_{m}^{*}\left[\left(s_{m} u_{m}\right) \delta_{m} u_{l}\right]\right\} \\
+\left(s_{k}(\tau \rho)\right)\left(s_{k} u_{k}\right) \delta_{k} u_{l}
\end{gathered}
$$

where $\eta=\eta(s \rho, s C)$ and $\zeta=\zeta(s \rho, s C)$ (or $\eta=s \eta(\rho, C)$ and $\zeta=s \zeta(\rho, C))$ are defined on $\omega_{h}^{*}$, with $s=s_{1} s_{2} s_{3}$.

For the spatial discretization (3.2)-(3.8), the following total mass and component mass conservation laws hold

$$
\partial_{t}(\rho, 1)=0, \partial_{t}(\rho C, 1)=0 \text { for } t \geq 0,
$$

cf. (2.8). They are easily obtained by taking the inner product in $H_{X}\left(\omega_{h}\right)$ of the balance equations (3.2) and (3.4) and 1 and applying the first formula (3.1).

Lemma 1. Let $\mathbf{u}, \mathbf{v} \in H_{X}\left(\omega_{h}\right)$, where $\mathbf{v}=\left(v_{1}, v_{2}, v_{3}\right)$. For the Navier-Stokes terms, the following self-adjointness and positive definiteness properties

$$
\begin{gathered}
\left(\Pi_{i j}^{N S}(\mathbf{u}), \delta_{i} v_{j}\right)_{i *}=\left(\delta_{i} u_{j}, \Pi_{i j}^{N S}(\mathbf{v})\right)_{i *}, \\
A^{N S}(\mathbf{u}):=\left(\Pi_{i j}^{N S}(\mathbf{u}), \delta_{i} u_{j}\right)_{i *} \\
\left.\geq\left(2 \eta,|\mathbf{D}(\mathbf{u})|_{h}^{2}\right)\right)_{*}+\left(\zeta-\frac{2}{3} \eta,\left(\operatorname{div}_{h} \mathbf{u}\right)^{2}\right)_{*} \geq 0,
\end{gathered}
$$

are valid, where

$$
\begin{aligned}
|\mathbf{D}(\mathbf{u})|_{h}^{2}=s_{2} s_{3}\left[\left(\delta_{1} u_{1}\right)^{2}\right]+s_{1} s_{3}\left[\left(\delta_{2} u_{2}\right)^{2}\right]+s_{1} s_{2}\left[\left(\delta_{3} u_{3}\right)^{2}\right] \\
+2 s_{3}\left(\mathbb{D}_{h 12}^{2}\right)+2 s_{2}\left(\mathbb{D}_{h 13}^{2}\right)+2 s_{1}\left(\mathbb{D}_{h 23}^{2}\right)
\end{aligned}
$$

with $\mathbb{D}_{h k l}=\frac{1}{2}\left(\delta_{l} s_{k} u_{k}+\delta_{k} s_{l} u_{l}\right)$ for $k \neq l$ and $\operatorname{div}_{h} \mathbf{u}=s_{2} s_{3} \delta_{1} u_{1}+s_{1} s_{3} \delta_{2} u_{2}+$ $s_{1} s_{2} \delta_{3} u_{3}$ on $\omega_{h}^{*}$.

This lemma is similar to [26, Lemma 3] (where boundary conditions were taken into account), and its proof is simpler.

The next main result of the section is a counterpart of Proposition 1.

Theorem 1. For the above spatial discretization (3.2)-(3.8), the following total energy inequality is satisfied

$$
\begin{aligned}
\partial_{t}\left(\rho \left(\Psi_{0}+\mathcal{E}_{\lambda}\right.\right. & \left.\left.+0.5|\mathbf{u}|^{2}-\Phi\right), 1\right)+A^{N S}(\mathbf{u}) \\
& +\left(M\left(s_{j} C\right) \delta_{j} \mu, \delta_{j} \mu\right)_{j *}+\left(\tau \rho \widetilde{w}_{j}^{(0)}, \widetilde{w}_{j}^{(0)}\right) \leq 0 \quad \text { for } t \geq 0,
\end{aligned}
$$

where $A^{N S}(\mathbf{u})$ is the Navier-Stokes contribution, see (3.9), and

$$
\begin{aligned}
\widetilde{w}_{k}^{(0)}:=s_{k}^{*}\left[\left(s_{k} u_{k}\right) \delta_{k} u_{k}+\delta_{k}\left(G_{h}-\Phi\right)\right. & \left.-\left(s_{k} \mu\right) \delta_{k} C\right] \\
& +s_{l}^{*}\left[\left(s_{l} u_{l}\right) \delta_{l} u_{k}\right]+s_{m}^{*}\left[\left(s_{m} u_{m}\right) \delta_{m} u_{k}\right]
\end{aligned}
$$


Consequently, the discrete total energy is non-increasing:

$$
\partial_{t}\left(\rho\left(\Psi_{0}+\mathcal{E}_{\lambda}+0.5|\mathbf{u}|^{2}-\Phi\right), 1\right) \leq 0 \text { for } t \geq 0
$$

Remark 1. The term $\left(\tau \rho \widetilde{w}_{1}^{(0)}, \tau \rho \widetilde{w}_{2}^{(0)}, \tau \rho \widetilde{w}_{3}^{(0)}\right)$ is an approximation for $\mathbf{m}$ on $\omega_{h}$, see (2.4), (2.9) and (2.10).

Proof. We first take the inner product in $H_{X}\left(\omega_{h}\right)$ of the mass balance equation $(3.2)$ and $\Psi_{1 \rho}^{\prime}-\Phi-0.5|\mathbf{u}|^{2}$. Using the first formula (3.1) and $0.5 \delta_{k}\left(v^{2}\right)=$ $\left(s_{k} v\right) \delta_{k} v$, we get

$$
\begin{aligned}
\left(\Psi_{1 \rho}^{\prime}, \partial_{t} \rho\right)-\partial_{t}(\rho \Phi, 1)- & 0.5\left(\partial_{t} \rho,|\mathbf{u}|^{2}\right) \\
& -\left(J_{i}, \delta_{i}\left(\Psi_{1 \rho}^{\prime}-\Phi\right)\right)_{i *}+\left(J_{i},\left(s_{i} u_{j}\right) \delta_{i} u_{j}\right)_{i *}=0
\end{aligned}
$$

Now we take the inner product in $H_{X}\left(\omega_{h}\right)$ of the momentum balance equation (3.3) and $u_{l}$ and use the formula

$$
\left[\partial_{t}\left(\rho u_{l}\right)\right] u_{l}=0.5 \partial_{t}\left(\rho u_{l}^{2}\right)+0.5\left(\partial_{t} \rho\right) u_{l}^{2} .
$$

Next we replace $l$ with $j$, sum up over $j=1,2,3$ and then apply formulas (3.1). Then we sum the obtained result and (3.11) (by doing so, the third and fifth terms on the left-hand side in (3.11) are canceled out) and obtain

$$
\begin{aligned}
& \left(\Psi_{1 \rho}^{\prime}, \partial_{t} \rho\right)+\partial_{t}\left(-\rho \Phi+0.5 \rho|\mathbf{u}|^{2}, 1\right)-\left(J_{j}, \delta_{j}\left(\Psi_{1 \rho}^{\prime}-\Phi\right)\right)_{j *}+A^{N S}(\mathbf{u}) \\
& +\left(\left(s_{j} \rho\right) \delta_{j}\left(G_{h}-\Phi\right), s_{j} u_{j}\right)_{j *}+\left(\Pi_{i j}^{\tau}, \delta_{i} u_{j}\right)_{i *}=\left(\left(s_{j} \mu\right)\left(s_{j} \rho\right) \delta_{j} C, s_{j} u_{j}\right)_{j *} .
\end{aligned}
$$

Owing to definitions of $J_{j}$ and $G_{h}$, see (3.6) and (3.5), we can rewrite the sum of the third and fourth terms on the left-hand side as

$$
\begin{aligned}
-\left(J_{j}, \delta_{j}\left(\Psi_{1 \rho}^{\prime}-\Phi\right)\right)_{j *}+\left(\left(s_{j} \rho\right) \delta_{j}\left(G_{h}-\Phi\right), s_{j} u_{j}\right)_{j *} & \\
& =\left(J_{j}, \delta_{j} \mathcal{E}_{\lambda}\right)_{j *}+\left(\mathrm{m}_{j}, \delta_{j}\left(G_{h}-\Phi\right)\right)_{j *} .
\end{aligned}
$$

Applying the formulas

$$
\partial_{t}(\rho C)=\rho \partial_{t} C+\left(\partial_{t} \rho\right) C, \quad \delta_{k}^{*}\left(J_{k} s_{k} C\right)=\left(\delta_{k}^{*} J_{k}\right) C+s_{k}^{*}\left(J_{k} \delta_{k} C\right)
$$

and the mass balance equation (3.2), we rewrite the component mass balance equation (3.4) in the following partially non-divergence form

$$
\rho \partial_{t} C+s_{i}^{*}\left(J_{i} \delta_{i} C\right)-\delta_{i}^{*}\left(M\left(s_{i} C\right) \delta_{i} \mu\right)=0 .
$$

Taking the inner product in $H_{X}\left(\omega_{h}\right)$ of it and $\mu=(1 / \rho)\left[\Psi_{1 C}^{\prime}-\delta_{i}^{*}\left(\lambda_{1}\left(s_{i} \rho\right) \delta_{i} C\right)\right]$, we derive

$$
\begin{aligned}
\left(\Psi_{1 C}^{\prime}, \partial_{t} C\right)-\left(\partial_{t} C, \delta_{i}^{*}(\right. & \left.\left.\lambda_{1}\left(s_{i} \rho\right) \delta_{i} C\right)\right) \\
& +\left(s_{i}^{*}\left(J_{i} \delta_{i} C\right), \mu\right)+\left(M\left(s_{i} C\right) \delta_{i} \mu, \delta_{i} \mu\right)_{i *}=0
\end{aligned}
$$


Using (3.1) and then the mass balance equation (3.2), we accomplish the following chain of transformations

$$
\begin{aligned}
& -\left(\partial_{t} C, \delta_{i}^{*}\left(\lambda_{1}\left(s_{i} \rho\right) \delta_{i} C\right)\right)=\left(\partial_{t} \delta_{i} C, \lambda_{1}\left(s_{i} \rho\right) \delta_{i} C\right)_{i *} \\
& \quad=\left(\lambda_{1}\left(s_{i} \rho\right), \frac{1}{2} \partial_{t}\left[\left(\delta_{i} C\right)^{2}\right]\right)_{i *}=\left(\rho, \lambda_{1} \frac{1}{2} \partial_{t} s_{i}^{*}\left[\left(\delta_{i} C\right)^{2}\right]\right) \\
& \quad=\left(\rho, \partial_{t} \mathcal{E}_{\lambda}\right)=\partial_{t}\left(\rho, \mathcal{E}_{\lambda}\right)-\left(\mathcal{E}_{\lambda}, \partial_{t} \rho\right)=\partial_{t}\left(\rho, \mathcal{E}_{\lambda}\right)-\left(\delta_{j} \mathcal{E}_{\lambda}, J_{j}\right)_{j *} .
\end{aligned}
$$

Applying again the second formula (3.1) and the formula $J_{l}=\left(s_{l} \rho\right) s_{l} u_{l}-\mathrm{m}_{l}$, from (3.14) we find

$$
\begin{aligned}
\left(\Psi_{1 C}^{\prime}, \partial_{t} C\right)+\partial_{t}\left(\rho, \mathcal{E}_{\lambda}\right) & -\left(\delta_{j} \mathcal{E}_{\lambda}, J_{j}\right)_{j *}+\left(\left(s_{i} \rho\right) s_{i} u_{i},\left(s_{i} \mu\right) \delta_{i} C\right)_{i *} \\
& -\left(\mathrm{m}_{i},\left(s_{i} \mu\right) \delta_{i} C\right)_{i *}+\left(M\left(s_{i} C\right) \delta_{i} \mu, \delta_{i} \mu\right)_{i *}=0 .
\end{aligned}
$$

Summing up equalities (3.12) and (3.15) and invoking (3.13), we obtain

$$
\begin{aligned}
\partial_{t}\left(\rho\left(\Psi_{0}+\mathcal{E}_{\lambda}+0.5|\mathbf{u}|^{2}-\Phi\right), 1\right)+ & A^{N S}(\mathbf{u}) \\
& +\left(M\left(s_{j} C\right) \delta_{j} \mu, \delta_{j} \mu\right)_{j *}+B^{\tau}=0,
\end{aligned}
$$

where the term $\left(\delta_{j} \mathcal{E}_{\lambda}, J_{j}\right)_{j *}$ and the term on the right-hand side of (3.12) have been canceled out and

$$
B^{\tau}:=\left(\mathrm{m}_{j}, \delta_{j}\left(G_{h}-\Phi\right)\right)_{j *}-\left(\mathrm{m}_{i},\left(s_{i} \mu\right) \delta_{i} C\right)_{i *}+\left(\Pi_{i j}^{\tau}, \delta_{i} u_{j}\right)_{i *} .
$$

It remains to transform and bound from below the term $B^{\tau}$. Similarly to $\left[27\right.$, Section 3.4], using the definition of $\Pi_{i j}^{\tau}$ we write down

$$
\begin{aligned}
B^{\tau}=\left(\mathrm{m}_{i},\left(s_{i} u_{i}\right) \delta_{i} u_{i}+\delta_{i}\left(G_{h}-\right.\right. & \left.\Phi)-\left(s_{i} \mu\right) \delta_{i} C\right)_{i *} \\
& +\left(1-\delta^{(i j)}\right)\left(\mathrm{m}_{i}^{(j)},\left(s_{i} u_{i}\right) \delta_{i} u_{j}\right)_{i *}=\delta^{(i i)} B_{i}^{\tau},
\end{aligned}
$$

where $\delta^{(i j)}$ is the Kronecker delta and

$$
\begin{aligned}
& B_{l}^{\tau}:=\left(\mathrm{m}_{l}, \widehat{\alpha}_{l l}\right)_{l *}+\left(\mathrm{m}_{l}^{(k)}, \alpha_{k l}\right)_{k *},+\left(\mathrm{m}_{l}^{(m)}, \alpha_{m l}\right)_{m *}, \\
& \widehat{\alpha}_{l l}:=\left(s_{l} u_{l}\right) \delta_{l} u_{l}+\delta_{l}\left(G_{h}-\Phi\right)-\left(s_{l} \mu\right) \delta_{l} C, \quad \alpha_{k l}:=\left(s_{k} u_{k}\right) \delta_{k} u_{l} .
\end{aligned}
$$

By virtue of definitions of $\mathrm{m}_{l}$ and $\mathrm{m}_{l}^{(k)}$, see (3.7) and (3.8), after transferring the operators $s_{l}, s_{k}$ and $s_{m}$ (see the second formula (3.1)) on the cofactors we get

$$
\begin{aligned}
B_{l}^{\tau}= & \left(s_{l}(\tau \rho) \widehat{\alpha}_{l l}+s_{l}\left[\tau \rho\left(s_{k}^{*} \alpha_{k l}+s_{m}^{*} \alpha_{m l}\right)\right], \widehat{\alpha}_{l l}\right)_{l *} \\
& +\left(s_{k}\left[\tau \rho\left(s_{l}^{*} \widehat{\alpha}_{l l}+s_{m}^{*} \alpha_{m l}\right)\right]+\left(s_{k}(\tau \rho)\right) \alpha_{k l}, \alpha_{k l}\right)_{k *} \\
& +\left(s_{m}\left[\tau \rho\left(s_{l}^{*} \widehat{\alpha}_{l l}+s_{k}^{*} \alpha_{k l}\right)\right]+\left(s_{m}(\tau \rho)\right) \alpha_{m l}, \alpha_{m l}\right)_{m *} \\
= & \left(\tau \rho, s_{l}^{*}\left(\widehat{\alpha}_{l l}^{2}\right)+s_{k}^{*}\left(\alpha_{k l}^{2}\right)+s_{m}^{*}\left(\alpha_{m l}^{2}\right)\right)+\left(\tau \rho\left(s_{k}^{*} \alpha_{k l}+s_{m}^{*} \alpha_{m l}\right), s_{l}^{*} \widehat{\alpha}_{l l}\right) \\
& +\left(\tau \rho,\left(s_{l}^{*} \widehat{\alpha}_{l l}+s_{m}^{*} \alpha_{m l}\right) s_{k}^{*} \alpha_{k l}+\left(s_{l}^{*} \widehat{\alpha}_{l l}+s_{k}^{*} \alpha_{k l}\right) s_{m}^{*} \alpha_{m l}\right) \\
= & \left(\tau \rho,\left(s_{l}^{*} \widehat{\alpha}_{l l}+s_{k}^{*} \alpha_{k l}+s_{m}^{*} \alpha_{m l}\right)^{2}+r\right)=\left(\tau \rho,\left(\widetilde{w}_{l}^{(0)}\right)^{2}+r\right),
\end{aligned}
$$


where (owing to the formula $\left.s_{l}^{*}\left(y^{2}\right)=\left(s_{l}^{*} y\right)^{2}+\frac{1}{4} h_{l}^{2}\left(\delta_{l}^{*} y\right)^{2}\right)$

$$
r=\frac{1}{4}\left[h_{l}^{2}\left(\delta_{l}^{*} \widehat{\alpha}_{l l}\right)^{2}+h_{k}^{2}\left(\delta_{k}^{*} \alpha_{k l}\right)^{2}+h_{m}^{2}\left(\delta_{m}^{*} \alpha_{m l}\right)^{2}\right] \geq 0 .
$$

Now the result of the theorem follows from (3.16).

Remark 2. Inequality (3.10) is valid for $\tau \geq 0$. If $\tau=0$, then it holds for the non-regularized NSCH equations (in this case, the term with $\tau$ disappears).

Remark 3. Similarly to [6], the term $s_{l}^{*}\left\{\left(s_{l} \rho\right)\left(s_{l} \mu\right) \delta_{l} C\right\}$ can be replaced with $\mu s_{l}^{*}\left[\left(s_{l} \rho\right) \delta_{l} C\right]$ in equation (3.3) together with replacing the term $\delta_{i}^{*}\left(J_{i} s_{i} C\right)$ with $\delta_{i}^{*}\left[J_{i} s_{i} C-(1 / 4) h_{i}^{2}\left(s_{i} \rho\right)\left(\delta_{i} C\right) \delta_{i} u_{i}\right]$ in equation (3.4). In this case, Theorem 1 remains valid. Actually, using the simple formula

$$
s_{k}\left(\mu u_{k}\right)=\left(s_{k} \mu\right) s_{k} u_{k}+\frac{1}{4} h_{k}^{2}\left(\delta_{k} \mu\right) \delta_{k} u_{k},
$$

we can complement the above proof with the following chain of equalities for $y_{l}:=\left(s_{l} \rho\right) \delta_{l} C$ :

$$
\begin{aligned}
\left(\mu s_{j}^{*} y_{j}, u_{j}\right) & =\left(y_{j}, s_{j}\left(\mu u_{j}\right)\right)_{j *}=\left(y_{j},\left(s_{j} \mu\right) s_{j} u_{j}\right)_{j *}+\left(y_{j}, \frac{1}{4} h_{j}^{2}\left(\delta_{j} \mu\right) \delta_{j} u_{j}\right)_{j *} \\
& =\left(\left(s_{j} \mu\right) y_{j}, s_{j} u_{j}\right)-\left(\delta_{j}\left[\frac{1}{4} h_{j}^{2} y_{j}\left(\delta_{j} u_{j}\right)\right], \mu\right) .
\end{aligned}
$$

2.3. Let us introduce the equilibrium solutions $\rho_{S}, C_{S} \in H_{X}\left(\omega_{h}\right)$ and $\mathbf{u}_{S}=0$ with $\rho_{S}>0$ and $0<C=C_{S}(x)<1$ to the above discretization (3.2)-(3.8).

Theorem 2. For $\tau_{0}=$ const $>0$, these equilibrium solutions satisfy the following equations

$$
\begin{aligned}
\Psi_{1 \rho}^{\prime}(\rho, C)+\frac{1}{2} \lambda_{1} s_{i}^{*}\left[\left(\delta_{i} C\right)^{2}\right]-\mu_{S} C-\Phi & \equiv G_{h}-\mu_{S} C-\Phi \equiv \text { const } \\
\Psi_{1 C}^{\prime}(\rho, C)-\delta_{i}^{*}\left(\lambda_{1}\left(s_{i} \rho\right) \delta_{i} C\right) & =\mu_{S} \rho, \quad \mu_{S} \equiv \text { const }
\end{aligned}
$$

on $\omega_{h}$, with the same functions $\Psi_{1}$ and $\Phi$ as in the differential case, see (2.12)(2.13).

Proof. For the equilibrium solutions, the following equations on $\omega_{h}$ hold

$$
\begin{aligned}
\delta_{i}^{*} J_{i S}=0, \quad s_{l}^{*}\left\{\left(s_{l} \rho\right)\left[\delta_{l}\left(G_{h}-\Phi\right)-\left(s_{l} \mu\right) \delta_{l} C\right]\right\} & =0, \\
\delta_{i}^{*}\left[J_{i S} s_{i} C-M\left(s_{i} C\right) \delta_{i} \mu\right] & =0,
\end{aligned}
$$

with $J_{l S}=\left(s_{l}\left(\tau_{0} \rho\right)\right)\left[\delta_{l}\left(G_{h}-\Phi\right)-\left(s_{l} \mu\right) \delta_{l} C\right], l=1,2,3$, corresponding to the differential equations (2.11). For $\tau_{0}=$ const $>0$, by virtue of equations (3.19) (the second one becomes $s_{l}^{*} J_{l S}=0, l=1,2,3$ ) we get

$$
\delta_{i}^{*}\left(J_{i S} s_{i} C\right)=\left(\delta_{i}^{*} J_{i S}\right) s_{i}^{*} s_{i} C+\left(s_{i}^{*} J_{i S}\right) \delta_{i}^{*} s_{i} C=0 .
$$

Therefore this term in equation (3.20) disappears, and its inner product in $H_{X}\left(\omega_{h}\right)$ with $\mu$ leads to the equality

$$
\left(M\left(s_{i} C\right),\left(\delta_{i} \mu\right)^{2}\right)_{i *}=0 .
$$


Thus $\delta_{i} \mu=0, i=1,2,3$, i.e., $\mu \equiv \mu_{S}=$ const.

Now, $J_{l S}=\tau_{0}\left(s_{l} \rho\right) \delta_{l}\left(G_{h}-\Phi-\mu_{S} C\right), l=1,2,3$, so that (similarly to [26, Section 2.2]) the inner product in $H_{X}\left(\omega_{h}\right)$ of the first equation (3.19) and $G_{h}-\Phi-\mu_{S} C$ results in

$$
\left(\tau_{0} s_{i} \rho,\left[\delta_{i}\left(G_{h}-\Phi-\mu_{S} C\right)\right]^{2}\right)_{* i}=0 .
$$

Consequently $\delta_{i}\left(G_{h}-\Phi-\mu_{S} C\right)=0, i=1,2,3$, i.e., $G_{h}-\Phi-\mu_{S} C \equiv$ const.

Eventually equations (3.19)-(3.20) are reduced to system (3.17)-(3.18) for $\rho_{S}$ and $C_{S}$ on $\omega_{h}$.

As in [26], the property established in Theorem 2 allows one to call the above discretization well-balanced similarly to the case of the shallow water equations, where the same property has long been discussed in the literature and an equation like (3.17) means that simply $g \rho_{S}-\Phi \equiv$ const on $\omega_{h}$, where $g$ is the gravitational constant. The presence of such a property is not guaranteed a priori and is provided only by the special construction of a discretization.

\section{Numerical experiments}

We apply the explicit Euler method for the approximation in time of both the above spatial discretization (3.2)-(3.8) and its modification from Remark 3 . We call $A$ and $B$ the respective full discretizations (finite-difference schemes). This section is devoted to their numerical investigation. We confine ourselves by the $2 \mathrm{D}$ case and consider $2 \mathrm{D}$ versions of these schemes for which a counterpart (simplification) of Theorem 1 remains valid.

In all problems below, we study the mixture of two components with the same state equations and thus with $c_{s 1}=c_{s 2}=c_{s}$. The following constants are in use $\eta=5 \cdot 10^{-4} \mathrm{~Pa} \cdot \mathrm{s}, \zeta=0, M=5 \cdot 10^{-8} \mathrm{~kg} \cdot \mathrm{s} / \mathrm{m}^{3}, c_{s}=1000 \mathrm{~m} / \mathrm{s}$, $A_{\psi}=10^{4} \mathrm{~J} / \mathrm{kg}$. The function $\Phi$, the parameter $\lambda_{1}$ and the initial functions $\rho_{0}$, $\mathbf{u}_{0}$ and $C_{0}$ are taken depending on the specific problem.

Also we choose the domain $\bar{\Omega}_{X}=\left[0, X_{1}\right] \times\left[0, X_{2}\right]$ with $X_{i}=X=0.01 \mathrm{~m}$ and the grid over it with $N_{i}=100$; then $h_{i}=h=10^{-4} \mathrm{~m}(i=1,2)$. The regularization parameter is associated with $h$ and is calculated as $\tau=0.5 h / c_{s}$. The stability issue in the linearized statement for the considered type of the regularization and approximation in time has recently been studied in detail in $1 \mathrm{D}$ and multiD cases in the one-component statement in $[28,29]$. Notice that we do not apply any regularization of the viscosity coefficient contrary to the usual QHD-approach.

Problem 3.1. We begin with a typical problem on merging of two droplets. Let $\Phi=0$ and $\lambda_{1}=6 \cdot 10^{-5} \mathrm{~J} \cdot \mathrm{m}^{2} / \mathrm{kg}$, and the initial data are as follows $\rho_{0}(x) \equiv 1, \mathbf{u}_{0}(x) \equiv \mathbf{0}$,

$$
C_{0}(x)=\left(1-2 \varepsilon_{c}\right) \frac{1}{2}\left\{2+\tanh \left[0.5 \beta\left(R-r_{1}(x)\right)\right]+\tanh \left[0.5 \beta\left(R-r_{2}(x)\right)\right]\right\}+\varepsilon_{c}
$$

with $x=\left(x_{1}, x_{2}\right), R=0.2 X, \beta=\sqrt{2 A_{\psi} / \lambda_{1}}$ and

$$
r_{1}(x)=|x-(0.3 X-h, 0.5 X)|, \quad r_{2}(x)=|x-(0.7 X+h, 0.5 X)|
$$




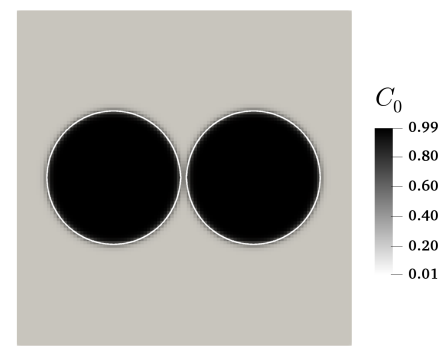

Figure 1. Problem 3.1: distribution of $C_{0}$. The white color indicates the isoline with $C=0.5$.

as well as $\varepsilon_{c}=0.01$ defining the "offset" of values $C_{0}$ from 0 and 1 , see Figure 1 .

The model parameters are connected with the surface tension coefficient $\sigma$ and the thickness of interface $l_{\varepsilon}$ (that is defined as the domain with $0.05 \leq$ $C \leq 0.95)$ by the formulas

$$
\sigma=\rho \sqrt{A_{\psi} \lambda_{1} / 18}, \quad l_{\varepsilon}=4.164 \sqrt{\lambda_{1} / A_{\psi}} .
$$

Actually they are approximate being obtained on the basis of solving the plane interface problem [18]. According to these formulas, we get $\sigma \approx 0.183 \mathrm{~J} / \mathrm{m}^{2}$ and $l_{\varepsilon} \approx 3.225 \cdot 10^{-4} \mathrm{~m} \approx 3.2 \mathrm{~h}$. We set the time step $\Delta t=3.2 \cdot 10^{-8} \mathrm{~s}$. Note that its value was first calculated theoretically in the linearized statement and then adjusted experimentally in order to increase it but not destroy the solution.

We define the discrete total energy and total kinetic energy

$$
E_{\text {tot }}:=\left(\rho\left(\Psi_{0}+\mathcal{E}_{\lambda}+0.5|\mathbf{u}|^{2}-\Phi\right), 1\right), \quad E_{\text {kin }}:=\left(0.5 \rho|\mathbf{u}|^{2}, 1\right)
$$

and compare results for schemes $A, B$ and scheme from [5] that we call scheme $C$. Scheme $C$ is conservative with respect to the momentum, and it was constructed to diminish imbalances in the total energy compared to schemes used previously. In its semi-discrete version, the general form of the discrete balance equations (3.2) and (3.4) remains the same but the discrete momentum balance equation differs from $(3.3)$ :

$$
\partial_{t}\left(\rho u_{l}\right)+\delta_{i}^{*}\left(J_{i} s_{i} u_{l}\right)+\stackrel{\circ}{\delta}_{l} p=\delta_{i}^{*}\left(\Pi_{i l}^{N S}-Q_{i l}^{(i)}+\Pi_{i l}^{\tau}\right)+s_{l}^{*}\left\{\left(s_{l} \rho\right) \delta_{l} \Phi\right\},
$$

which corresponds to the original momentum equation (2.2). Here $\stackrel{\circ}{\delta}_{l}=\delta_{l}^{*} s_{l}=$ $s_{l}^{*} \delta_{l}$ is the central difference quotient and, in the $2 \mathrm{D}$ case,

$$
Q_{k k}^{(k)}=\lambda_{1} s_{k}\left\{\left[s_{k}^{*}\left(\left(s_{k} \rho\right) \delta_{k} C\right)\right] \stackrel{\circ}{\delta_{k} C}\right\}, Q_{k l}^{(k)}=\lambda_{1} s_{l}^{*}\left\{\left[s_{l}\left(\left(s_{k} \rho\right) \delta_{x} C\right)\right] s_{k} \delta_{l} C\right\}, k \neq l,
$$

see (2.6). The formulas for $J_{i}, \Pi_{i l}^{N S}$ and $\mu$ are the same as above but the expression for $\mathrm{m}_{k}$ is different, in the $2 \mathrm{D}$ case, as follows:

$$
\begin{aligned}
\mathrm{m}_{k}= & \left(s_{k} \tau\right)\left(s_{k} \rho\right)\left[\left(s_{k} u_{k}\right) \delta_{k} u_{k}+\left(s_{k} u_{l}\right) \stackrel{\circ}{\delta_{l}} s_{k} u_{k}-\delta_{k} \Phi\right] \\
& +\left(s_{k} \tau\right)\left(\delta_{k} p+\delta_{k} Q_{k k}+\delta_{l}^{*} Q_{l k}^{(k, l)}\right), k \neq l,
\end{aligned}
$$




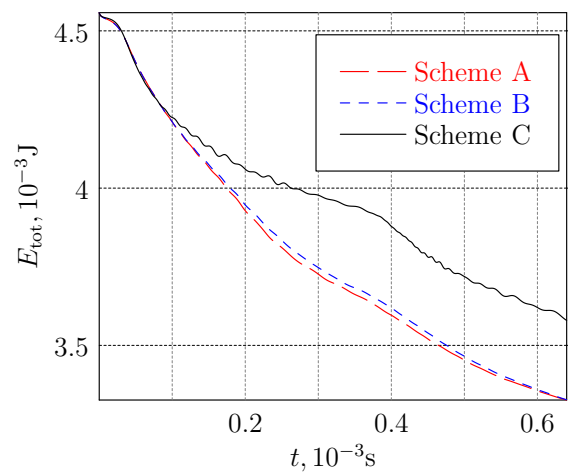

Figure 2. Problem 3.1: plots of $E_{\text {tot }}(t)$ for the schemes for $\lambda_{1}=6 \cdot 10^{-5} \mathrm{~J} \cdot \mathrm{m}^{2} / \mathrm{kg}$.

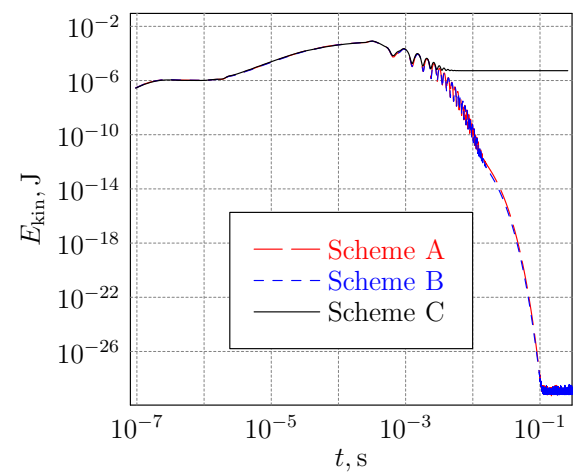

Figure 3. Problem 3.1: plots of $E_{\text {kin }}(t)$ for the schemes for $\lambda_{1}=2 \cdot 10^{-4} \mathrm{~J} \cdot \mathrm{m}^{2} / \mathrm{kg}$.

see (2.4). Here formulas for $\Pi_{i l}^{\tau}, Q_{k k}$ and $Q_{l k}^{(k, l)}$ are omitted for brevity.

In Figure 2, the plots of $E_{\text {tot }}(t)$ for schemes $A, B$ and $C$ are presented. For scheme $C$, the total energy oscillates with a moderate amplitude (so that it is not non-increasing) and decays significantly slower then for schemes $A$ and $B$.

We also take the larger value $\lambda_{1}=2 \cdot 10^{-4} \mathrm{~J} \cdot \mathrm{m}^{2} / \mathrm{kg}$ (the rest parameters are the same). Then according to (4.1) we have $\sigma \approx 0.33 \mathrm{~J} / \mathrm{m}^{2}$ and $l_{\varepsilon} \approx 5.9 h$. For such $\lambda_{1}$, the function $E_{\text {tot }}(t)$ becomes monotone for all three schemes. But the behavior of $E_{\text {kin }}(t)$ differs qualitatively: for schemes $A$ and $B$, it decreases down to computer zero whereas, for scheme $C$ and $t$ large enough, it stays almost constant $\left(5.87 \cdot 10^{-3} \mathrm{~J}\right)$, see Figure 3 . The last fact indicates the presence of the spurious (parasitic) currents mentioned in Introduction.

For illustrative purposes, the distribution of $|\mathbf{u}|$ for scheme $C$ and large $t$ is presented in Figure 4a. It is clear that in the vicinity of interface $|\mathbf{u}|$ is significantly greater than zero. In Figure $4 \mathrm{~b}$, the quarter of numerical domain is presented, where arrows correspond to $\mathbf{u}$ (their length is proportional to $|\mathbf{u}|$ ) and the black thin and thick lines represent respectively the stream lines and the isoline $C=0.5$. Notice that schemes $A$ and $B$ are free of spurious currents in the sense that the maximum value of $|\mathbf{u}|$ over domain is zero within the computer precision when the solution stabilizes.

Distributions of $C$ and $\rho$ do not differ significantly for all the schemes. Moreover, for schemes $A$ and $B$ they almost coincide. In Figures $5 \mathrm{a}$ and $5 \mathrm{~b}$, they are presented for $x_{1} \in[0.5 X, X]$ and $x_{2}=0.5 X$ in the vicinity of interface. In Figure 5b, clearly the density values inside and outside the droplet are respectively $\rho_{\text {in }}=1.0001473 \mathrm{~kg} / \mathrm{m}^{3}$ and $\rho_{\text {out }}=1.0000290 \mathrm{~kg} / \mathrm{m}^{3}$. This small difference is due to the interphase (surface) tension which is connected to the droplet radius by the known Laplace law

$$
\Delta p \equiv p_{\text {in }}-p_{\text {out }}=\sigma_{L} / R,
$$

where $\Delta p=c_{s}^{2}\left(\rho_{\text {in }}-\rho_{\text {out }}\right)$ by virtue of the state equation and $R$ is the droplet radius which equals $\approx 28 h=2.8 \cdot 10^{-3} \mathrm{~m}$. In accordance with (4.2), we have $\sigma_{L}=0.331145 \mathrm{~J} / \mathrm{m}^{2}$ that is quite close to $\sigma$ obtained from the first formula (4.1). It is important here that we deal with compressible fluids in this paper. 


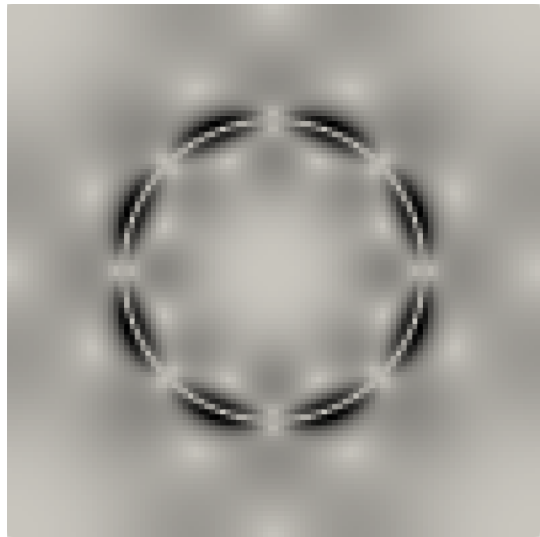

(a) Distribution of $|\mathbf{u}|$. Lightgrey and black colors correspond to $10^{-5} \mathrm{~m} / \mathrm{s}$ and $1.3 \mathrm{~m} / \mathrm{s}$ respectively.

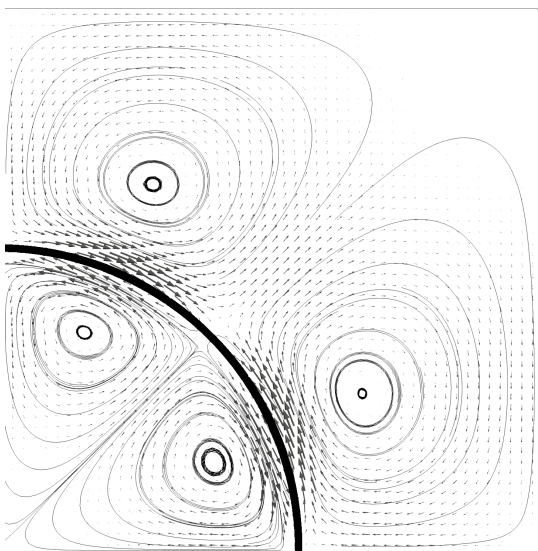

(b) Distribution of $\mathbf{u}$ and stream lines in the quarter of numerical domain.

Figure 4. Problem 3.1: distributions of $|\mathbf{u}|$ and $\mathbf{u}$ for $t=4 \cdot 10^{6} \Delta t=0.19 \mathrm{~s}$ for scheme $C$. An impact of spurious currents is observed.

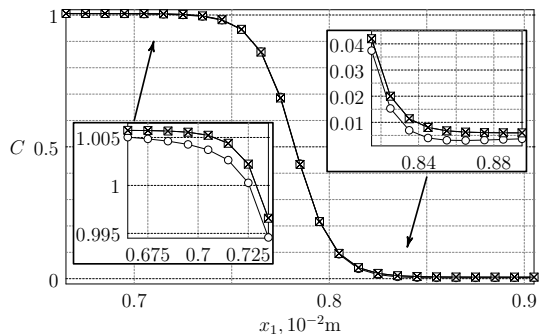

(a) Distribution of $C$.

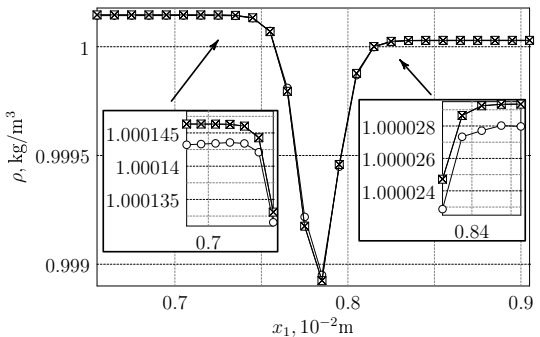

(b) Distribution of $\rho$.

Figure 5. Problem 3.1: distributions of $C$ and $\rho$ for $x_{1} \in[0.5 X, X]$ and $x_{2}=0.5 X$ in the vicinity of interface for $t=4 \cdot 10^{6} \Delta t=0.19 \mathrm{~s}$. The symbols $\square, \times$ and $\circ$ correspond to schemes $A, B$ and $C$ respectively.

Also notice that there are values of $C$ slightly greater than 1 in Figure 5a. Sometimes this phenomenon is called "the bulk shift" in literature, for example, see [12]. As mentioned in [9, 12,23,24], this is due to using formula (2.7) for $\Psi_{\text {sep }}(C)$. The formula is simple and convenient, but it does not have singularities as $C \rightarrow+0$ and $C \rightarrow 1-0$ (that would be more relevant physically).

It is significant that during the numerical simulation by schemes $A$ and $B$ the total momentum of the system $(\rho \mathbf{u}, 1)$ remains close to $\approx 10^{-17}-10^{-20}$ (the algorithms were implemented using $\mathrm{C}++$ programming language, compiler g++ 7.4.0 and double-precision variables). Therefore, despite of the formal lack of conservativeness with respect to the momentum for schemes $A$ and $B$, clearly the total momentum is conserved up to the computer precision.

Notice that the less the surface tension effect and better resolution of the 


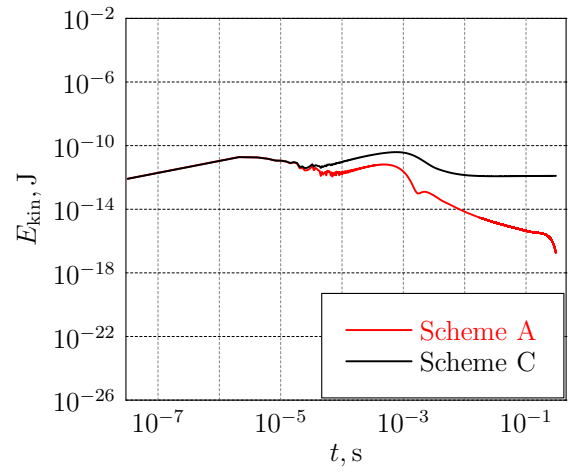

(a)

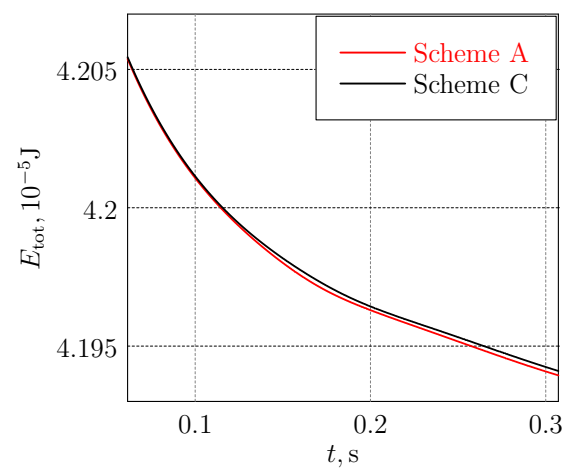

(b)

Figure 6. Problem 3.1: plots of $E_{\mathrm{kin}}(t)$ (a) and $E_{\mathrm{tot}}(t)$ (b) for schemes $A$ and $C$ in the case $\lambda_{1}=4 \cdot 10^{-6} \mathrm{~J} \cdot \mathrm{m}^{2} / \mathrm{kg}$ and $A_{\psi}=0.5 \cdot 10^{2} \mathrm{~J} / \mathrm{kg}$.

interface take place, the less the results obtained by schemes $A$ and $C$ differ. Figure 6 a shows the dependencies $E_{\text {kin }}(t)$ obtained by them when setting the parameters $\lambda_{1}=4 \cdot 10^{-6} \mathrm{~J} \cdot \mathrm{m}^{2} / \mathrm{kg}$ and $A_{\psi}=0.5 \cdot 10^{2} \mathrm{~J} / \mathrm{kg}$ corresponding to the values $\sigma \approx 0.0033 \mathrm{~J} / \mathrm{m}^{2}$ and $l_{\varepsilon} \approx 11.8 h$. Quantitatively, their behavior differs much less than previously for much larger $\sigma \approx 0.33 \mathrm{~J} / \mathrm{m}^{2}$, see Figure 3. But qualitatively the behavior is similar, and, for scheme $C$, the parasitic currents still present though now the corresponding dependencies $E_{\text {tot }}(t)$ are very close, moreover, both of them are decreasing, for $t \in[0.06,0.3] \mathrm{s}$, see Figure $6 \mathrm{~b}$. Also the distributions of $C$ and $\rho$ (which we omit here) coincide visually for both schemes $A$ and $C$.

Note that schemes $A$ and $B$ lead to practically the same results for the sufficiently smooth initial data.

Finally, we analyze the case where the regularization is absent, i.e., $\tau=0$. In Figure 7, we present plots of $E_{\text {tot }}(t)-\tilde{E}$, with $\tilde{E}=4.224599 \cdot 10^{-5} \mathrm{~J}$, obtained by scheme $A$ for the main $\tau=0.5 h / c_{s}$ versus $\tau=0$, for the above time step $\Delta t$ and much less time step $\Delta t / 10$. We observe that their behavior is similar only for very small times but then, for $\tau=0, E_{\text {tot }}(t)$ grows rapidly rather than decreases. This process is faster for the time step $\Delta t$ and slightly slower for the time step $\Delta t / 10$ but ultimately there is no significant difference, and the both solutions are even destroyed.

Problem 3.2. The next problem deals with the initially stationary droplet of radius $R$ influenced by $x_{2}$-periodic body force defined by the non-zero potential

$$
\Phi\left(x_{2}\right)=\tilde{g} \cos \left(\frac{2 \pi}{X} x_{2}\right), \quad \tilde{g}=7 \cdot 10^{5} \mathrm{~m} \cdot \mathrm{N} / \mathrm{kg} .
$$

We set the initial data as follows

$$
\begin{aligned}
\rho_{0}(x) & =\exp \left\{\Phi\left(x_{2}\right) / c_{s}^{2}\right\}, \quad \mathbf{u}_{0}(x)=\mathbf{0} \\
C_{0}(x) & =\left(1-2 \varepsilon_{c}\right) 0.5\{1+\tanh [0.5 \beta(R-r(x))]\}+\varepsilon_{c}
\end{aligned}
$$




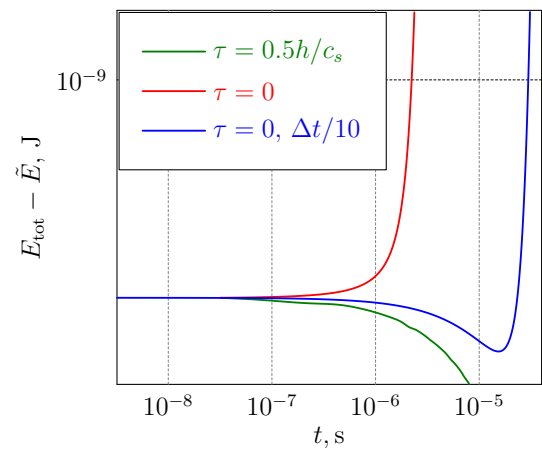

Figure 7. Problem 3.1: plots of $E_{\text {tot }}(t)$ obtained by scheme $A$ for the main $\tau$ and $\tau=0$ and two time steps.

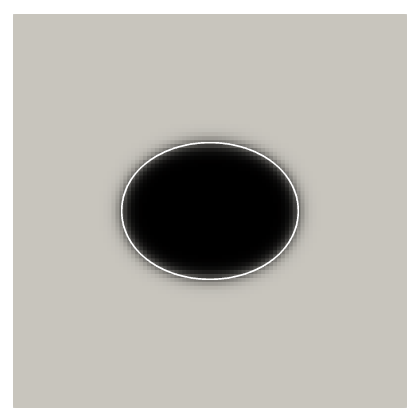

Figure 8. Problem 3.2: distribution of $C$ for $t=3.5 \cdot 10^{6} \Delta t$ obtained by scheme $A$.

where $\varepsilon_{c}=0.01, R=0.2 X$ and $r(x)=|x-0.5(X, X)|$. The form of $\rho_{0}(x)$ corresponds to its distribution in the homogeneous single-component fluid (without considering $C$ ) influenced by the body force with the given potential. We set $\lambda_{1}=2 \cdot 10^{-4} \mathrm{~J} \cdot \mathrm{m}^{2} / \mathrm{kg}$ and the time step $\Delta t=2 \cdot 10^{-8} \mathrm{~s}$.

The distribution of $C$ for $t=3.5 \cdot 10^{6} \Delta t$ computed by scheme $A$ is presented in Figure 8 where the white color indicates the contour $C=0.5$. The initially circular-shaped droplet is squeezed along $x_{2}$ direction by the body force. A similar shape is obtained by scheme $C$ but it produces the slightly thinner interface. More significantly, for scheme $A$ and $B$ the total energy $E_{\text {tot }}(t)$ is non-increasing and kinetic energy goes to zero (once again the results are almost identical for both schemes). On the contrary, for scheme $C$, the total energy is not monotone and the kinetic energy stays almost constant $\left(3.98 \cdot 10^{-5} \mathrm{~J}\right)$ from $t \approx 10^{-4} \mathrm{~s}$, see Figures $9 \mathrm{a}$ and $9 \mathrm{~b}$.

In this problem, for schemes $A$ and $B$, the total momentum is conserved up to the values $\sim 10^{-14}$ that is worse than in the previous problem but still quite acceptable.

Problem 3.3. Finally, we present results of numerical simulation by scheme $A$ of a droplet at the equilibrium state. These results are in accordance with the Laplace law for the $2 \mathrm{D}$ case $(4.2)$. Here we assume that $\rho_{\text {in }}$ and $\rho_{\text {out }}$ are the average density values inside and outside the droplet. The value of $\rho_{\text {in }}$ is computed by averaging over grid cells with $C \geq 0.5+\tilde{\varepsilon}_{c}$ (i.e., the cells inside the droplet and at some distance from the interface). Similarly, to compute $\rho_{\text {out }}$, the cells with $C \leq 0.5-\tilde{\varepsilon}_{c}$ are taken. Here $\tilde{\varepsilon}_{c}$ is a small parameter.

We set $\Phi=0$ and $\lambda_{1}=2 \cdot 10^{-4} \mathrm{~J} \cdot \mathrm{m}^{2} / \mathrm{kg}$. According to the first (approximate) formula (4.1), the surface tension coefficient equals $\sigma=0.33 \mathrm{~J} / \mathrm{m}^{2}$. Also we take $\rho_{0}(x) \equiv 1 \mathrm{~kg} / \mathrm{m}^{3}, \mathbf{u}_{0}=\mathbf{0}$ and $C_{0}$ as in the previous problem. In simulations, the different values of droplet radius $R$ are used.

The dependence of $\Delta p$ on the computed (observable) radius $R_{a}$ is presented in Figure 10. Here $R_{a}=h \sqrt{N_{C>0.5} / \pi}$, where $N_{C>0.5}$ is the number of cells with $C>0.5$. In simulation results, we get that $R_{a}$ is very close to its initial value $R$. Note that, for $\tilde{\varepsilon}_{c}=0.01$, the pressure outside the droplet cannot be 


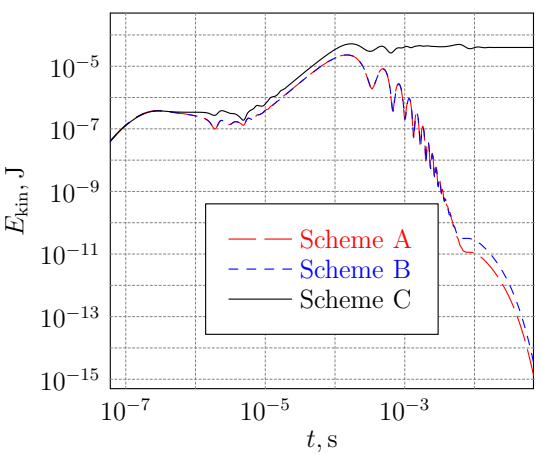

(a) $E_{\mathrm{kin}}(t)$

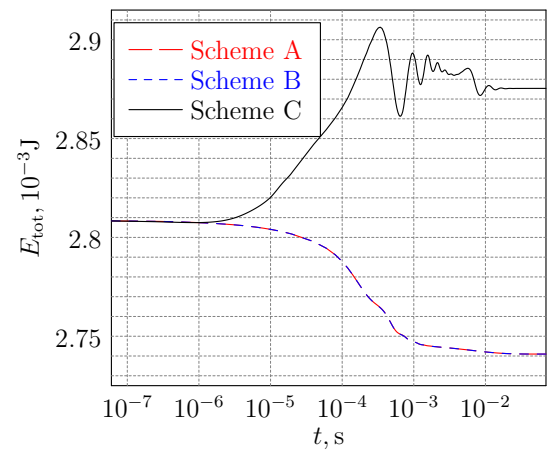

(b) $E_{\text {tot }}(t)$

Figure 9. Problem 3.2: plots of $E_{\text {kin }}(t)$ and $E_{\text {tot }}(t)$ for schemes $A, B$ and $C$ obtained for the body force potential $\Phi$ of form (4.3).

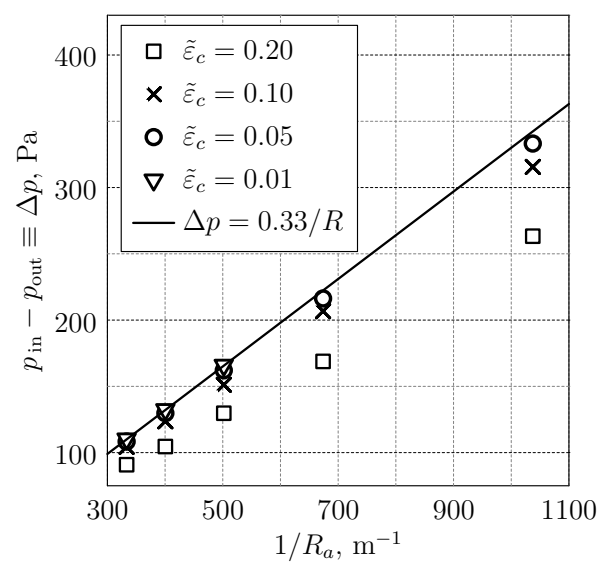

Figure 10. Problem 3.3: dependence of the computed pressure drop $\Delta p$ on the computed droplet radius $R_{a}$ for different $\tilde{\varepsilon}_{c}$.

computed for $R=15 h, 10 h$ since the minimal (over the whole computational domain) value of $C$ is greater than 0.01. As mentioned above, the reason of that is "the bulk shift" effect, see Figure 5a, since $C$ increasing (over the whole domain) is proportional to the Cahn number $\mathrm{Cn}=l_{\varepsilon} / R$ according to $[12,24]$. In Figure 10, clearly the results closest to the theoretical ones (for all studied radii) are those obtained for $\tilde{\varepsilon}_{c}=0.05$.

\section{Acknowledgements}

The study was supported by the Russian Science Foundation, Project No. 1911-00169. 


\section{References}

[1] M.O. Abu-Al-Saud, S. Popinet and H.A. Tchelepi. A conservative and well-balanced surface tension model. J. Comput. Phys., 371:896-913, 2018. https://doi.org/10.1016/j.jcp.2018.02.022.

[2] D.M. Anderson, G.B. McFadden and A.A. Wheeler. Diffuse-interface methods in fluid mechanics. Ann. Rev. Fluid Mech., 30(1):139-165, 1998. https://doi.org/10.1146/annurev.fluid.30.1.139.

[3] V. Balashov and E. Savenkov. Quasi-hydrodynamic model of multiphase fluid flows taking into account phase interaction. J. Appl. Mech. Tech. Phys., 59(3):434-444, 2018. https://doi.org/10.1134/S0021894418030069.

[4] V. Balashov, E. Savenkov and A. Zlotnik. Numerical method for 3D two-component isothermal compressible flows with application to digital rock physics. Russ. J. Numer. Anal. Math. Model., 34(1):1-13, 2019. https://doi.org/10.1515/rnam-2019-0001.

[5] V. Balashov, A. Zlotnik and E. Savenkov. Analysis of a regularized model for the isothermal two-component mixture with the diffuse interface. Russ. J. Numer. Anal. Math. Model., 32(6):347-358, 2017. https://doi.org/10.1515/rnam-20170033 .

[6] V.A. Balashov and E.B. Savenkov. Thermodynamically consistent spatial discretization of the one-dimensional regularized system of the Navier-Stokes-CahnHilliard equations. J. Comput. Appl. Math., (in press).

[7] B.N. Chetverushkin. Kinetic Schemes and Quasi-Gasdynamic System of Equations. CIMNE, Barcelona, 2008.

[8] K. Connington and T. Lee. A review of spurious currents in the lattice Boltzmann method for multiphase flows. J. Mech. Sci. Technology, 26(12):3857-3863, 2012. https://doi.org/10.1007/s12206-012-1011-5.

[9] M.I.M. Copetti and C.M. Elliott. Numerical analysis of the Cahn-Hilliard equation with a logarithmic free energy. Numer. Math., 63(1):39-65, 1992. https://doi.org/10.1007/BF01385847.

[10] T.G. Elizarova. Quasi-Gas Dynamic Equations. Springer, Berlin-Heidelberg, 2009.

[11] T.G. Elizarova, A.A. Zlotnik and B.N. Chetverushkin. On quasi-gasdynamic and quasi-hydrodynamic equations for binary gas mixtures. Dokl. Math., 90(3):719723, 2014. https://doi.org/10.1134/S1064562414070217.

[12] F. Frank, C. Liu, F.O. Alpak and B. Riviere. A finite volume/discontinuous Galerkin method for the advective Cahn-Hilliard equation with degenerate mobility on porous domains stemming from micro-CT imaging. Comput. Geosci., 22(2):543-563, 2018. https://doi.org/10.1007/s10596-017-9709-1.

[13] J.-L. Guermond and B. Popov. Viscous regularization of the Euler equations and entropy principles. SIAM J. Appl. Math., 74(2):284-305, 2014. https://doi.org/10.1137/120903312.

[14] D.J.E. Harvie, M.R. Davidson and M. Rudman. An analysis of parasitic current generation in volume of fluid simulations. Appl. Math. Model., 30(10):1056-1066, 2006. https://doi.org/10.1016/j.apm.2005.08.015.

[15] D. Jacqmin. Calculation of two-phase Navier-Stokes flows using phase-field modeling. J. Comput. Phys., 155(1):96-127, 1999. https://doi.org/10.1006/jcph.1999.6332. 
[16] D. Jamet, D. Torres and J.U. Brackbill. On the theory and computation of surface tension: the elimination of parasitic currents through energy conservation in the second-gradient method. J. Comput. Phys., 182(1):262-276, 2002. https://doi.org/10.1006/jcph.2002.7165.

[17] J. Kim. A continuous surface tension force formulation for diffuse-interface models. J. Comput. Phys., 204(2):784-804, 2005. https://doi.org/10.1016/j.jcp.2004.10.032.

[18] J. Liu. Thermodynamically consistent modeling and simulation of multiphase flows. PhD thesis, The University of Texas at Austin, 2014.

[19] J. Lowengrub and L. Truskinovsky. Quasi-incompressible Cahn-Hilliard fluids and topological transitions. Proc. Royal Soc. London. A., 454:2617-2654, 1998. https://doi.org/10.1098/rspa.1998.0273.

[20] N. Provatas and K. Elde. Phase-field methods in material science and engineering. Weinheim: Willey-VCH, 2010.

[21] Yu. V. Sheretov. Continuum dynamics under spatiotemporal averaging. R\&C Dynamics, Moscow-Izhevsk, 2009. (in Russian)

[22] M. Svärd. A new Eulerian model for viscous and heat conducting compressible flows. Phys. A: Stat. Mech. Appl., 506:350-375, 2018. https://doi.org/10.1016/j.physa.2018.03.097.

[23] G. Tierra and F. Guillén-González. Numerical methods for solving the CahnHilliard equation and its applicability to related energy-based models. Arch. Comput. Meth. Eng., 22(2):269-289, 2015. https://doi.org/10.1007/s11831-0149112-1.

[24] P. Yue, C. Zhou and J.J. Feng. Spontaneous shrinkage of drops and mass conservation in phase-field simulations. J. Comput. Phys., 223(1):1-9, 2007. https://doi.org/10.1016/j.jcp.2006.11.020.

[25] A. Zlotnik. On the energy dissipative spatial discretization of the barotropic quasi-gasdynamic and compressible Navier-Stokes systems of equations in polar coordinates. Russ. J. Numer. Anal. Math. Model., 33(3):199-210, 2018. https://doi.org/10.1515/rnam-2018-0017.

[26] A.A. Zlotnik. On conservative spatial discretizations of the barotropic quasi-gasdynamic system of equations with a potential body force. Comput. Math. Math. Phys., 56(2):303-319, 2016. https://doi.org/10.1134/S0965542516020160.

[27] A.A. Zlotnik. Entropy-conservative spatial discretization of the multidimensional quasi-gasdynamic system of equations. Comput. Math. Math. Phys., 57(4):706725, 2017. https://doi.org/10.1134/S0965542517020166.

[28] A.A. Zlotnik and T.A. Lomonosov. Conditions for $L^{2}$-dissipativity of linearized explicit difference schemes with regularization for 1D barotropic gas dynamics equations. Comput. Math. Math. Phys., 59(3):452-464, 2019. https://doi.org/10.1134/S0965542519030151.

[29] A.A. Zlotnik and T.A. Lomonosov. On $L^{2}$-dissipativity of a linearized explicit finite-difference scheme with QGD-regularization for the barotropic gas dynamics system of equations. Dokl. Math., (in press). 